\title{
ESCOLIOSIS Y SÍNDROME DE PRADER-WILLI: A PROPÓSITO DE 5 CASOS INTERVENIDOS QUIRÚRGICAMENTE
}

\author{
ESCOLIOSE E SÍNDROME DE PRADER-WILLI: ANÁLISE DE CINCO CASOS \\ TRATADOS CIRURGICAMENTE
}

SCOLIOSIS AND PRADER-WILLI SYNDROME: REVIEW OF FIVE CASES

TREATED SURGICALLY

Jose Grass Pedrals ${ }^{1}$, Karen Weissmann Marcuson ${ }^{2}$, Veronica Herrera Gallegos ${ }^{3}$

\begin{abstract}
RESUMEN
Objetivo: Analizar los resultados y las complicaciones del tratamiento quirúrgico de la escoliosis, en pacientes portadores del Síndrome de Prader-Willi, para verificar si se justifica este tipo de tratamiento. Métodos: Análisis retrospectivo de las fichas clínicas y radiografías de los cinco pacientes portadores del Síndrome de Prader-Willi que consultaron en el Departamento de Escoliosis entre los años 2005 y 2010 , y fueron operados. Resultados: La edad de la consulta inicial fue en promedio 3,6 años; el promedio de escoliosis fue $61^{\circ}$, el que aumentó a $65^{\circ}$ al momento de la cirugía. El promedio de operaciones realizadas fue 2,8 por paciente, lográndose una corrección promedio de $35 \%$. Los motivos de las reintervenciones fueron pérdida de corrección en 4 casos (28,5\%), aflojamiento de ganchos en 2 (14,3\%) y xifosis referente a la instrumentación en uno $(7,1 \%)$ No hubo complicaciones graves. Conclusiones: Aún cuando la literatura muestra autores poco proclives a indicar cirugía en este tipo de pacientes, por la elevada tasa de complicaciones graves, la ausencia de ellas en nuestro medio hace mantener válidos los mismos criterios quirúrgicos que en escoliosis idiopática.
\end{abstract}

Descriptores: Síndrome de Prader-Willi; Escoliosis, Cirugía; Escoliosis, Complicaciones.

\section{RESUMO}

Objetivo: Analisar os resultados e as complicações do tratamento cirúrgico da escoliose em pacientes com síndrome de Prader-Willi para verificar se esse tipo de tratamento é justificado. Métodos: Análise retrospectiva dos prontuários clínicos e das radiografias dos cinco pacientes portadores de síndrome de Prader-Willi tratados cirurgicamente no Departamento de Escoliose entre 2005 e 2010 . Resultados: A média de idade dos pacientes na consulta inicial foi 3,6 anos. A média de escoliose foi $61^{\circ}$, a qual tinha aumentado para $65^{\circ}$ na ocasião da cirurgia. A média de cirurgias realizadas foi 2,8 por paciente, atingindo-se correção média de 35\%. Os motivos das re-intervenções foram perda de correção em quatro casos (28,5\%), afrouxamento dos ganchos em dois (14,3\%) e cifose depois da instrumentação em um caso (7,1\%). Não foram verificadas complicações graves. Conclusões: Mesmo que a literatura mostre autores pouco inclinados a indicar cirurgia nesse tipo de pacientes, por causa da elevada taxa de complicações graves, a ausência dessas complicações em nossa prática valida os mesmos critérios cirúrgicos da escoliose idiopática.

Descritores: Síndrome de Prader-Willi; Escoliose, Cirurgia; Escoliose, Complicações.

\begin{abstract}
Objective: Analyze the results and complications of scoliosis surgery in patients with Prader-Willi syndrome to verify if this kind of treatment is justified. Methods: Retrospective analysis of clinical records and radiographs of five patients with Prader-Willi syndrome treated at the Department of Scoliosis between 2005 and 2010. Results: The average age of patients at the first visit was 3.6 years. The average scoliosis was $61^{\circ}$, which increased to $65^{\circ}$ at the time of surgery. The average number of surgeries performed per patient was 2.8 , reaching an average correction of 35\%. The reasons for re-interventions were correction loss in 4 cases (28.5\%), loosening of hooks in two cases (14.3\%), and kyphosis after instrumentation in one case (7.1\%). There were no serious complications. Conclusions: Even though the literature shows authors unwilling to indicate surgery in such patients because of the high rate of severe complications, the absence of these complications in our practice validates the use of the same surgical criteria of idiopathic scoliosis.
\end{abstract}

Keywords: Prader-Willi syndrome; Scoliosis, Surgery; Scoliosis, Complications.

\section{INTRODUCCION}

El Síndrome de Prader Willi es una enfermedad genética descrita por primera vez en 1956 por Prader et al' ${ }^{1}$. Sus manifestaciones clínicas incluyen, apetito insaciable, obesidad, retardo mental e inmadurez sexual .La prevalencia es de aproximadamente 1 por 25.000 habitantes y es la principal causa genética de obesidade severa².

El Síndrome es de aparición esporádica y el 70\% de los casos ocurre por deleción del cromosoma 15, de origen paterno ${ }^{3}$, causa que no aumenta la frecuencia de escoliosis con respecto a quienes no presentan dicha anormalidad. cromosómica 4 .

Las alteraciones musculo-esqueléticas comienzan con hipotonía neonatal severa ${ }^{2}$, retraso importante en el inicio de la marcha ${ }^{3}$, laxitud ligamentosa, anormalidad de extremidades, displasia de cadera ${ }^{5}$ y osteoporosis. Un $40 \%$ de los casos presenta disminución

1. Jefe de Servicio de Traumatologia, Hospital Luis Calvo Mackenna y Profesor Asistente, Facultad de Medicina Universidad de Chile - Santiago, Chile.

2. Instructor, Facultad de Medicina Universidad del Desarrollo - Santiago, Chile.

3. Medico del Servicio deTraumatologia, Hospital Luis Calvo Mackenna - Santiago, Chile.

Trabajo realizado en Hospital Luis Calvo Mackenna - Santiago, Chile.

Correspondência: Jose Grass, E. mail :jgrass@vtr.net 
de los reflejos osteo-tendíneos profundos ${ }^{2}$, que no se correlaciona con ninguna alteración ortopédica. La escoliosis esta presente entre el $15 \%$ y el $86 \%$ de los pacientes ${ }^{5-7}$.

Si bien la obesidad es la manifestación característica, un 42\% puede no presentarla ${ }^{2}$ y su presencia o ausencia no determina la asociación a alteraciones esqueléticas.

En 1993 Holms et al. ${ }^{8}$ publicó los criterios diagnósticos, que son aceptados por los especialistas.

En vista de la poca frecuencia de consulta de ese tipo de pacientes en los Servicios de Traumatología y la percepción, apoyada por la literatura, de que estos casos presentan mas dificultades técnicas y riesgo quirúrgico que las escoliosis idiopáticas, decidimos revisar y describir los casos que consultaron en nuestro Servicio y fueron operados por escoliosis

\section{MATERIAL Y METODOS}

Se realiza un análisis retrospectivo de 5 pacientes portadores del Sindrome de Prader Willi, confirmado de acuerdo a los criterios de Holms et al. ${ }^{8}$ derivados por deformidad de columna al Departamento de Escoliosis del Servicio de Traumatología del Hospital Luis Calvo Mackenna, entre los años 2005 y 2010. El análisis incluyó la revisión de la ficha clínica y las radiografias de columna completa de pié, pre y post operatorias, tanto antero-posterior como lateral .

Las tecnicas quirurgica empleadas fueron de tres tipos:

1. Artrodesis anterior mediante toracotomia, resección discal y colocación de injerto costal intervertebral

2. Hemiatrodesis, que consistió en abordar sólo el lado convexo de la curva, respetando procesos espinosos y efectuando decorticacion y destrucción de articulares. Esta técnica incluyó instrumentacion con una barra y ganchos multiples en los procesos transversos, aplicando fuerza contractora (Figuras 1 y 2 ).

3. Artrodesis bilateral clásica, consistente en destruccion de articulares y decorticación a ambos lados de la curva e instrumentación con ganchos múltilples sub laminares, supralaminares y pediculares, conectados a dos barras y estabilizados con dos cross link. Las fuerzas aplicadas fueron desrotación de la barra en $90^{\circ}$ con la técnica de Cotrel Dubousset clássica, asociado a contracción del lado convexo (Figura 3).

Tanto la hemi-artrodesis como la artrodesis bilateral incluyeron uso de injerto autólogo, suplementado con injero óseo halógeno de banco.

Todas las cirugías se efectuaron con hemodilución e hipotensión controlada de PAM entre 60 y $65 \mathrm{~mm}$. El instrumental empleado fue, en todos los casos, de titánio (Ti6Al4V).
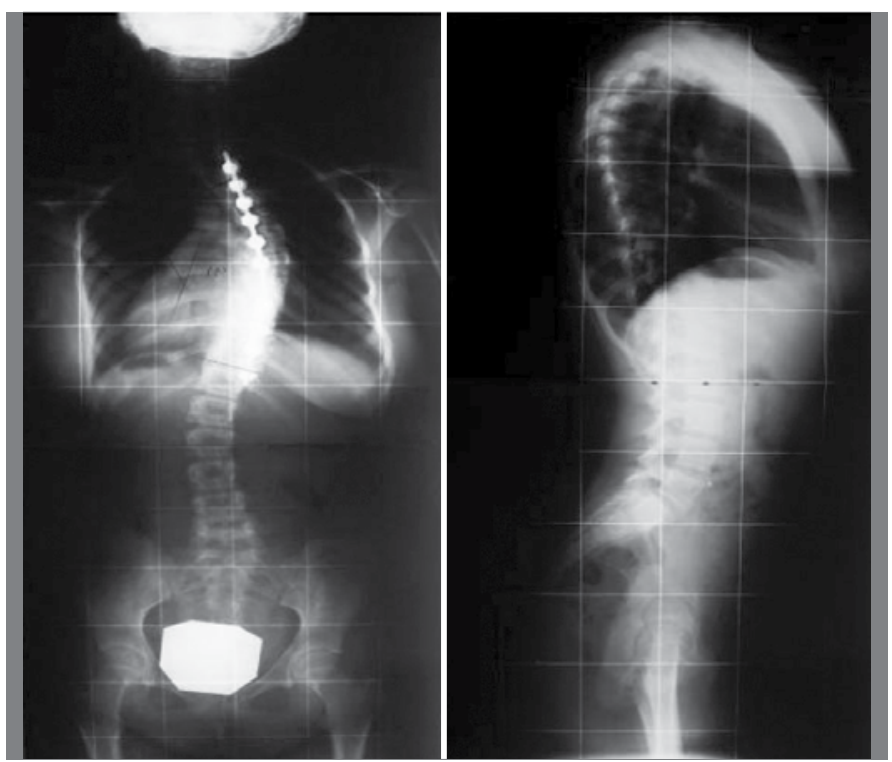

Figura 1. Radiografías preoperatorias de uno de los casos.

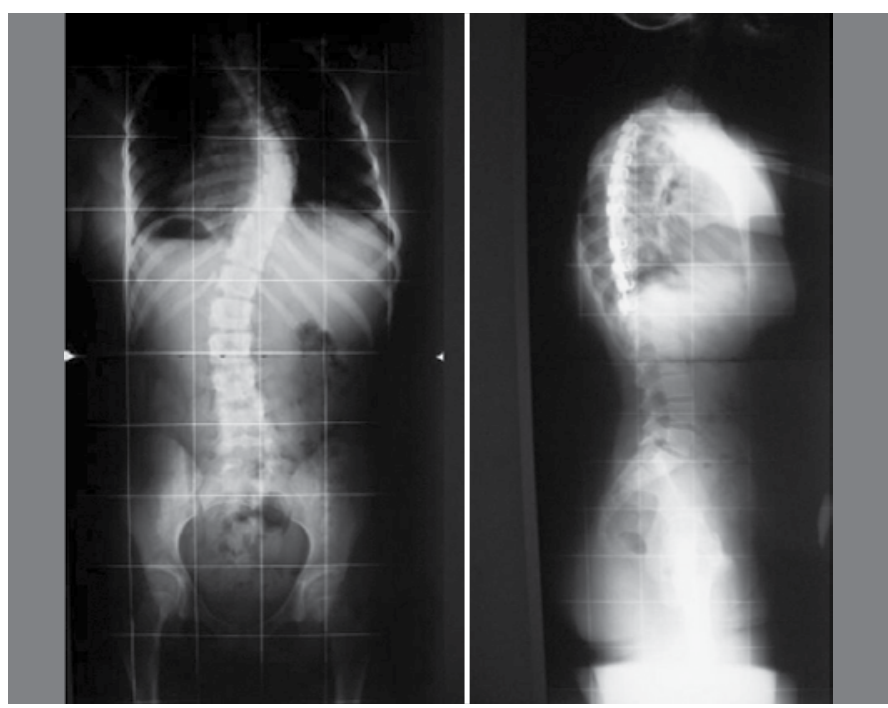

Figura 2. Radiografías post hemiartrodesis posterior instrumentada del mismo caso.

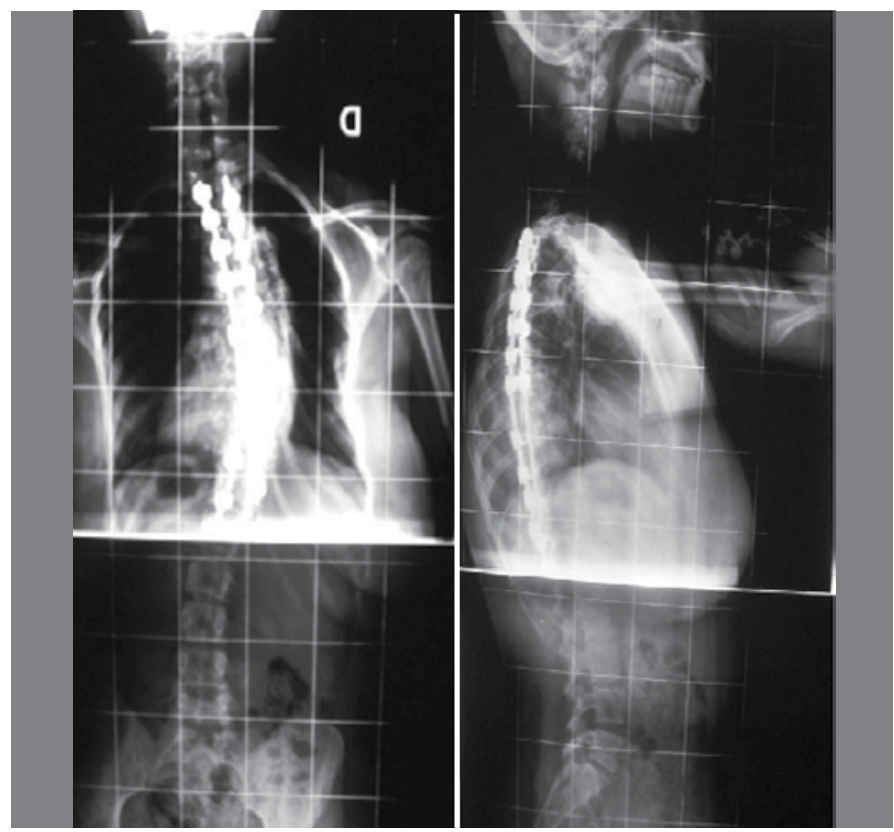

Figura 3. Radiografías post artrodesis posterior definitiva del mismo caso.

\section{RESULTADOS}

La edad en la primera consulta fue entre 2.6 y 15.9 años, con un promedio de 3.6 años. Al momento de ésta, se confeccionó la ficha clínica y se indicó toma de radiografías de columna. La magnitud de la escoliosis encontrada varió entre $45^{\circ}$ y $83^{\circ}$ (Tabla 1), con un promedio de $61^{\circ}$ y una mediana de $53^{\circ}$. En tres pacientes, con curvas flexibles, se indicó inicialmente corset ortopédico, el que fue usado en forma irregular por un promedio de 2 años y finalmente se indicó cirugía por intolerancia al corset y agravamento. Dos pacientes con curvas rígidas superiores a $70^{\circ}$ recibieron indicación de cirugía desde el primer momento.

Una vez indicada la cirugía, el tiempo de espera para la realización de ésta fue entre 1 y 14 meses, con un promedio de 8 meses. El estudio preoperatorio incluyó evaluación por cardiólogo, bronco-pulmonar, neurólogo y anestesista

Al momento de efectuarse la cirugía la escoliosis tenía un promedio de $65^{\circ}$, una mediana de $53^{\circ}$, con un rango de $43^{\circ}$ a $103^{\circ}$ (Figura 4) y la xifosis promedio era de $51^{\circ}$, con una mediana de $45^{\circ}$ (rango de $43^{\circ}$ a $71^{\circ}$ ). 
Tabla 1. Datos preoperatorios.

\begin{tabular}{c|c|c|c|c}
\hline Caso & Sexo/edad dignóstico & Angulo escoliosis & Angulo xifosis & Tipo curva \\
\hline 1 & $\mathrm{M} / 2,6$ años & $\mathrm{T} 4-\mathrm{T} 10: 52^{\circ}$ & $\mathrm{T} 2-\mathrm{L} 1: 43^{\circ}$ & Torácica \\
\hline 2 & $\mathrm{~F} / 2,8$ años & $\mathrm{T} 3-\mathrm{L} 3: 72^{\circ}$ & $\mathrm{T} 2-\mathrm{L} 2: 45^{\circ}$ & Toraco-lumbar \\
\hline 3 & $\mathrm{~F} / 2,11$ años & $\mathrm{T} 4-\mathrm{L} 2: 83^{\circ}$ & $\mathrm{T} 2-\mathrm{L} 2: 55^{\circ}$ & Toraco-lumbar \\
\hline 4 & $\mathrm{~F} / 10,3$ años & $\mathrm{T} 2-\mathrm{T} 10: 53^{\circ}$ & $\mathrm{T} 4-\mathrm{L} 1: 34^{\circ}$ & Torácica \\
\hline 5 & $\mathrm{~F} / 15,9$ años & $\mathrm{T} 5-\mathrm{T} 10: 45^{\circ}$ & $\mathrm{T} 2-\mathrm{T} 12: 68^{\circ}$ & Torácica \\
\hline
\end{tabular}

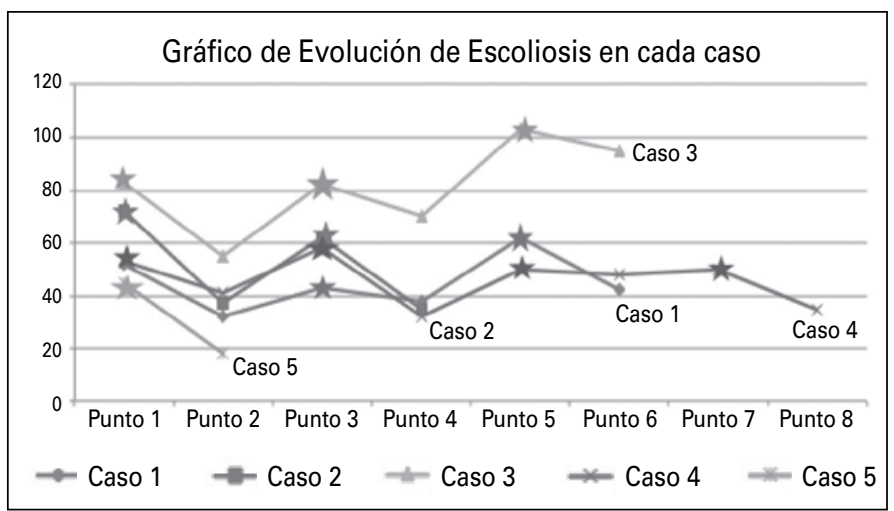

Figura 4. Evolucion en grados de escoliosis para cada paciente.

Cada estrella representa un evento quirúrgico.

De los 5 pacientes, sólo un paciente de 16 años (Figura 5) fue sometido a una artrodesis posterior como primera y única cirugia. Los otros cuatro, todos menores de 11 años fueron sometidos inicialmente a una hemi-artrodesis y posteriormente se agregó una artrodesis anterior por estar presentando un fenómeno del cigueñal (kranshaft). En un caso, ante un agravamento, se efectuó retoque de la hemiártrodesis, cruenteando el callo óseo y agregando más injerto. En otro caso, el retoque se asoció a prolongación de la instrumentación por sumación de nuevas vértebras a la curva.

En total, para los cinco pacientes incluídos en el estudio, se realizaron entre 1 y 4 cirugías (promedio 2.8). En 4 casos la causa de re-operación fué perdida de corrección y fenómeno del cigueñal, en otros 2 fue el aflojamiento de implantes de los extremos y en otro caso, la aparición de xifosis sobre la instrumentación.

Al momento de la artrodesis definitiva por vía posterior, se intentó efectuar monitoreo intra-operatorio de Potenciales Evocados motores y sensitivos en todos los pacientes. Sin embargo, el procedimiento sólo se logró en forma completa en 2 pacientes. En un tercero, sólo fue posible obtener registro sensitivo. En dos pacientes no fue posible obtener ningún registro, por no contarse con agujas de longitud concordante con la capa de tejido adiposo de las extremidades y el estado neurológico se evaluó con la técnica de Stagnara, durante las maniobras correctoras

Todos los pacientes fueron sometidos a profilaxis antibiótica con una dosis de cefazolina EV durante la inducción anestésica, que fué repetida a las $6 \mathrm{hrs}$

El sangrado intra-operatorio fluctuó entre 100 cc y 400 cc, con un promedio de 230 cc, ningun caso requirió transfusión de sangre.

El tiempo quirúrgico promedio fue de 2 horas 15 minutos, fluctuando entre 1 hora 10 minutos y 3 horas 20 minutos. Ningún paciente requirió UTI para manejo del postoperatorio. El Promedio de hospitalización, en las 14 cirugías efectuadas, fue de 5.4 días

No se presentaron complicaciones neurológicas ni respiratorias. Sólo un caso presentó dehiscencia de la herida operatoria a los 5 días de la cirugia, sin signos de infección y con cultivo negativo. Se trató con re-sutura, sin uso de antibióticos

El promedio de corrección obtenida en las 14 cirugías de los 5 pacientes incluídos en este estúdio, fue de 35\% (rango entre 4 y 48\%).

El promedio de corrección al momento del último control, en relación a la peor angulación que presentó cada paciente, fue de $45 \%$, con un rango de $34 \%$ a $60 \%$ (Figura 4).

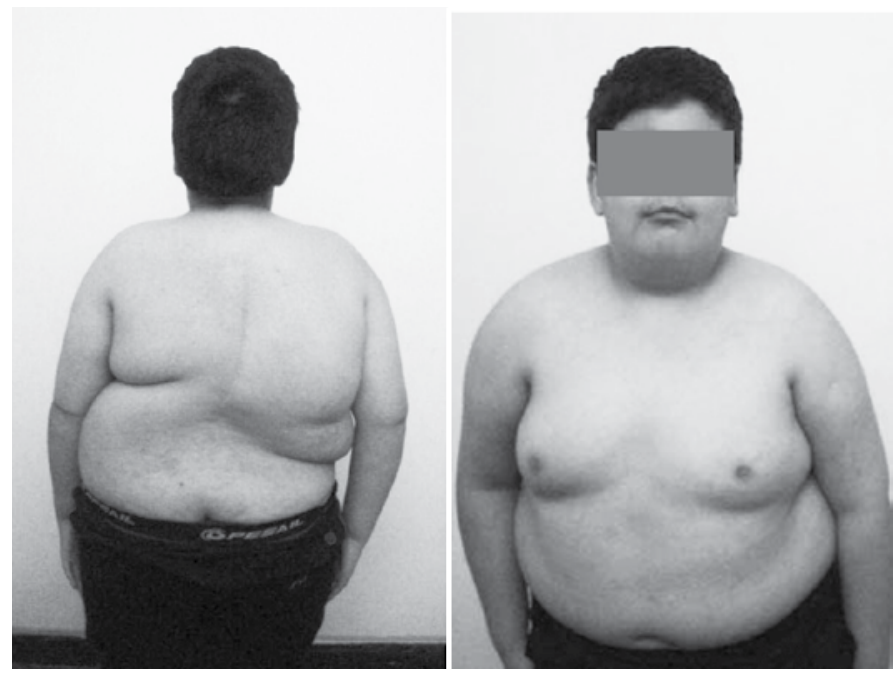

Figura 5. Paciente de 16 años, único caso sometido a una sola cirugía por via posterior.

\section{DISCUSION}

Las manifestaciones músculo esqueléticas del Sindrome de Prader Willi fueron descritas inicialmente por Pearson et al. ${ }^{9}$, descripción que incluyó dismetría de extremidades, escoliosis, coxa valga y manos o pies pequeños. Publicaciones posteriores agregaron mal alineamiento de extremidades y claudicación de marcha $(2,5)$, pie valgo, genu recurvatum, inestabilidad patelar y metatarso aducto ${ }^{10}$.

Un 17\% de los pacientes portadores de este Sindrome requieren cirugía ortopédica, generalmente como tratamiento de fracturas ${ }^{2}$ que afectan al $42 \%$ de los portadores del Sindrome $^{5}$ y que se pueden presentar hasta cuatro veces por paciente. Esto parece estar influído por la osteopenia y disminución de sensibilidade al dolor. En nuestra serie ningún paciente requirió someterse a cirugía de extremidades por causa ortopédica o traumática y tampoco ningun paciente tenía antecedentes de fracuras previas.

La literatura reporta que en pacientes con esta afección, la escoliosis se presenta entre un $15 \%$ y un $86 \%^{2,11,12}$, siendo la frecuencia mayor a mayor edad ${ }^{13}$. No podemos estabelecer prevalencia de escoliosis entre los portadores de este Sindrome en Chile, dado que se refieren al Departamento de Escoliosis sólo aquellos portadores de deformidad de columna y no existen estadísticas nacionales.

Al momento del diagnóstico de escoliosis, el 44\% de los pacientes presenta curvas sobre $20^{013}$ y sólo el $10 \%$ sobre los $40^{014}$, siendo generalmente niños mayores de 12 años ${ }^{15}$. En nuestros pacientes, extrañamente fueron los pacientes de menor edad quienes presentaron ángulos iniciales mayores, es asi como el caso de 3.3 años tenía $83^{\circ}$ y el de 6.6 años tenía $72^{\circ}$ (Tabla 1).

El diagnóstico temprano de escoliosis en nuestros pacientes, en promedio a los 3.6 años, es tambien inferior al descrito en la literatura, en que es en promedio a los 6 años $^{16}$. Esto puede deberse a que el Sistema de Salud Pública chileno permite un expedito acceso al especialista.

Ninguno de nuestros pacientes recibió algun tipo de terapia con hormona del crecimiento, tratamento de uso frecuente en pacientes con esta patología y cuyo objetivo es cambiar la presentación clínica, en cuanto a composición de masa corporal ${ }^{7,17}$. Esto se basa en que este Sindrome tiene manifestaciones clínicas comunes con el Sindrome de déficit de hormona del crecimiento, como son: disminución de masa corporal, crecimiento lento, aumento de tejido graso y disminución de la densidad mineral ósea ${ }^{7}$. En muchos de estos pacientes también se ha comprobado una menor producción de dicha hormona ${ }^{18}$. Los efectos de la terapia hormonal en la evolución de la escoliosis son controvertidos, algunos autores reportan efectos negativos ${ }^{7,12,17}$, mientras otras series lo encuentran beneficioso ${ }^{4,7}$. 
En Chile hay escasas publicaciones sobre el Sindrome de Prader Willi y sólo abordan el tratamento quirúrgico de la obesidad ${ }^{19}$, el uso de hormonas de crecimiento ${ }^{18} \mathrm{o}$ el estudio genético ${ }^{20}$.

Nuestros pacientes fueron sometidos a un promedio de 2.8 cirugías. Las causas de las re intervenciones han sido: pérdida de corrección, sumación de vértebras a la curva y aflojamiento de implantes. En la literatura, no sólo se describe una alta frecuencia de reintervenciones, sino también una alta frecuencia de complicaciones mayores ${ }^{16,21}$,como la paraplegia, por lo que muchos autores son muy consevadores en las indicaciones de cirugía.

Entre los pocos reportes de tratamento quirúrgico de escoliosis está Greggi et al... ${ }^{21}$, quien describe 6 casos con curvas entre $65^{\circ}$ y $96^{\circ}$ operadas con diferentes métodos. El $50 \%$ de sus casos presentó complicaciones que incluyeron paraplejia, aflojamiento de instrumentación y xifosis sobre instrumentación. Accadblet et al. ${ }^{16}$ conserva las indicaciones habituales de cirugía en curvas mayores a $40^{\circ}$, pero tambien presenta complicaciones en 9 de 16 pacientes, que incluyen 4 xifosis sobre la instrumentación, 2 infecciones profundas, 1 paraplejia, 1 pseudoartrosis y 1 retardo de la cicatrización de la herida. Al re-operar las 4 xifosis, tres presentaron paraplejia definitiva, por lo que el $25 \%$ de sus pacientes operados quedó con daño neurológico severo.

En una revisión de la base de datos Pubmed ${ }^{22}$, se encontraron sólo 5 publicaciones que evalúan el tratamento quirúrgico, de un total de 210 trabajos cuyo tema era Prader Willi. Por ser tan alta la tasa de complicaciones y mortalidad, se propone restingir la cirugía para casos con curvas mayores a $70^{\circ}$, mas aun cuando algunas series pequenas postulan que las curvas se estabilizarían al alcanzar los $70^{\circ 15}$, por lo que no se justificaria efectuar cirugías de tan alto riesgo.

La frecuencia de xifosis en los niveles cefálicos a la instrumentación, que afectó a uno de nuestros pacientes y motivó una re intervención, es atribuída a las características especiales de esta enfermedad, que incluye laxitud ligamentosa, debilidad muscular y tendencia natural a tener la cabeza hacia adelante ${ }^{5}$.

La disminución de la lordosis toráxica y la presencia de xifosis cervico-dorsal ,se describe en un $48 \%{ }^{5}$.
En relación a la localización de la escoliosis, la literatura describe que el $62 \%$ es toraco-lumbar o lumbar; el $28 \%$ doble curva y el 10\% toráxica 4 . Pero en nuestra serie la curva toráxica se presentó en un $60 \%$ y los otros casos fueron de localización toraco-lumbar.

Los reportes señalan que entre el $20 \%$ y el $44 \%$ de los pacientes tienen curvas mayores a $20^{\circ 13,23}$, por lo que pudieran requerir tratamento ortopédico y sólo el 10\% tiene curvas sobre los $40^{\circ 14}$, por lo que requerirían tratamento quirúrgico, siendo la mayoría dobles curvas. Como no tenemos datos del universo del que salen nuestros pacientes, no podemos estabelecer frecuencias. La prevalencia de escoliosis publicada es de $30 \%$ en menores de 10 años y $80 \%$ en adolescentes ${ }^{13}$. Algunos autores describen asociación entre escoliosis y mal alineamiento de extremidades o sexo femenino ${ }^{2}$.

Los tratamientos, sean con corset o quirúrgicos, son de difícil manejo en esta afección, por las características propias del síndrome, que incluye obesidad, retardo mental, difícil control de los impulsos, actitud desafiante, disminución de la sensibilidad al dolor y osteoporosis ${ }^{5}$.

El tratamento quirúrgico en este tipo de pacientes es técnicamente laborioso y presenta complicaciones intra y post operatorias que la distinguen de la escoliosis idiopática. En nuestros pacientes la elección de hemi-artrodesis como técnica quirúrgica más frecuente (6 de 14 cirugías) disminuyó los tiempos quirúrgicos y el hecho de haber asociado artrodesis anterior en 4 de los 5 pacientes debe haber influído en la obtención de una fusión mas sólida.

\section{CONCLUSIONES}

A pesar de la alta frecuencia de complicaciones descritas en la literatura, creemos que la cirugía debe ser indicada precozmente en este tipo de pacientes y en los de menor edad la hemi-artrodesis es una buena alternativa como tratamento inicial , para permitir un mayor crecimiento de tronco. Cuande llega el momento de la cirugía definitiva, la asociación a artrodesis anterior favorece la obtención de resultados duraderos.

\section{REFERENCIAS}

1. Parder A, Labhart A, Willi H. A syndrome with adiposity, stunted growth, cryptocordia and oligophrenia after myotonia entitled in newborn. Schweiz Med Wochenschr. 1956;86:1260-1.

2. Butler MG. Prader-Willi syndrome: current understanding of cause and diagnosis. Am J Med Genet. 1990;35(3):319-32.

3. Fridman C, Varela MC, Kok F, Setian N, Koiffmann CP. Prader-Willi syndrome: genetic tests and clinical findings. Genet Test. 2000:4(4):387-92.

4. Nakamura Y, Nagai T, lida T, Ozeki S, Nohara Y. Epidemiological aspects of scoliosis in a cohort of Japanese patients with Prader-Willi syndrome. Spine J. 2009;9(10):809-16.

5. Kroonen LT, Herman M, Pizzutillo PD, Macewen GD. Prader-Willi Syndrome: clinical concerns for the orthopaedic surgeon. J Pediatr Orthop. 2006;26(5):673-9.

6. West LA, Ballock RT. High incidence of hip dysplasia but not slipped capital femoral epiphysis in patients with Prader-Willi syndrome. J Pediatr Orthop. 2004;24(5):565-7.

7. de Lind van Wijngaarden RF, de Klerk LW, Festen DA, Duivenvoorden HJ, Otten BJ, et al. Randomized controlled trial to investigate the effects of growth hormone treatment on scoliosis in children with Prader-Willi syndrome. J Clin Endocrinol Metab. 2009;94(4):1274-80.

8. Holm VA, Cassidy SB, Butler MG, Hanchett JM, Greenswag LR, Whitman BY, et al. Prader-Willi syndrome: consensus diagnostic criteria. Pediatrics. 1993;91(2):398-402.

9. Pearson KD, Steinbach HL, Bier DM. Roentgenographic manifestations of the Prader-Willi syndrome. Radiology. 1971;100(2):369-77.

10. Nyunt O, Gupta A, Harris M, Walsh J, Cotterill AM. Orthopaedic manifestations of children with Prader-Willi Syndrome. In: $7^{\text {th }}$ Scientific Conference International Prader Willi Syndrome Organization. Taipei, Taiwan, May 20-21, 2010.

11. Odent T, Accadbled F, Koureas G, Cournot M, Moine A, Diene G, et al. Scoliosis in patients with Prader-Willi Syndrome. Pediatrics. 2008;122(2):e499-503.

12. Butler JV, Whittington JE, Holland AJ, Boer H, Clarke D, Webb T. Prevalence of, and risk factors for, physical ill-health in people with Prader-Willi syndrome: a population-based study. Dev Med Child Neurol. 2002;44(4):248-55.

13. de Lind van Wijngaarden RF, de Klerk LW, Festen DA, Hokken-Koelega AC. Scoliosis in Prader-Willi syndrome: prevalence, effects of age, gender, body mass index, lean body mass and genotype. Arch Dis Child. 2008;93(12):1012-6.

14. Nagai T, NakamuraY, Kakihana M, Obata K, Sakazume S, Murakami Ni. Frequency and risk factors of severe scoliosis in Prader-Willi Syndrome. In: 7th Scientific Conference International Prader Willi Syndrome Organization. Taipei, Taiwan, May 20-21, 2010.

15. Weiss HR, Bohr S. Conservative scoliosis treatment in patients with Prader-Willi syndrome. Stud Health Technol Inform. 2008;140:314-7.

16. Accadbled F, Odent T, Moine A, Chau E, Glorion C, Diene G, et al. Complications of scoliosis surgery in Prader-Willi syndrome. Spine (Phila Pa 1976). 2008 15;33(4):394-401.

17. Day GA, McPhee IB, Batch J, Tomlinson FH. Growth rates and the prevalence and progression of scoliosis in short-statured children on Australian growth hormone treatment programmes. Scoliosis. 2007;2:3.

18. Youlton R. [Prader-Willi syndrome. Treatment with growth hormone in 2 cases]. Rev Med Chil. 2001;129(10):1186-90

19. Braghetto I, Rodríguez A, Debandi A, Brunet L, Papapietro K, Pineda Pet AL. [Prader Willi Syndrome (PWS) associated to morbid obesity: surgical treatment]. Rev Med Chil. 2003;131(4):427-31.

20. Cortes F, Unanue N, Franco G, Velaszquez P, Horsthemke B. Prader Willi Syndrome Phenotype in a Chilean Patient with A 15011-013 Tetrasomy of Maternal Origin. In: 7th Scientific Conference International Prader Willi Syndrome Organization. Taipei, Taiwan, May 20-21, 2010.

21. Greggi T, Martikos K, Lolli F, Bakaloudis G, Di Silvestre M, Cioni A, et al. Treatment of scoliosis in patients affected with Prader-Willi syndrome using various techniques. Scoliosis. 2010 15:5:11.

22. Weiss HR, Goodall D. Scoliosis in patients with Prader Willi Syndrome - comparisons of conservative and surgical treatment. Scoliosis. 2009;4:10.

23. Holm VA, Laurnen EL. Prader-Willi syndrome and scoliosis. Dev Med Child Neurol. $1981 ; 23(2): 192-201$. 